\title{
Spinal arteriovenous metameric syndrome
}

INSERM

\section{Source}

INSERM. (1999). Orphanet: an online rare disease and orphan drug data base. Spinal arteriovenous metameric syndrome. ORPHA:53721

Cobb syndrome is defined by the association of vascular cutaneous (venous or arteriovenous), muscular (arteriovenous), osseous (arteriovenous) and medullary (arteriovenous) lesions at the same metamere or spinal segment. This segmental distribution may involve one or many of the 31 metameres present in humans. Only 16\% of the medullary lesions are multiple and have a clearly metameric distribution. 